\title{
IMAGE DEBLURRING BASED ON SPECTRAL MEASURES OF WHITENESS
}

\author{
Swathi and Bhoopathy Bagan \\ Department of Electronics Engineering, Madras Institute of Technology, \\ Anna University, Chennai, India
}

\begin{abstract}
Image Deblurring is an ill-posed inverse problem used to reconstruct the sharp image from the unknown blurred image. This process involves restoration of high frequency information from the blurred image. It includes a learning technique which initially focuses on the main edges of the image and then gradually takes details into account. As blind image deblurring is ill-posed, it has infinite number of solutions leading to an ill-conditioned blur operator. So regularization or prior knowledge on both the unknown image and the blur operator is needed to address this problem. The performance of this optimization problem depends on the regularization parameter and the iteration number. In already existing methods the iterations have to be manually stopped. In this paper, a new idea is proposed to regulate the number of iterations and the regularization parameter automatically. The proposed criteria yields, on average, an ISNR only 0.38dB below what is obtained by manual stopping. The results obtained with synthetically blurred images are good and considerable, even when the blur operator is ill-conditioned and the blurred image is noisy.
\end{abstract}

\section{KEYWORDS}

Image deconvolution, blind image deblurring, Image restoration, whiteness measures

\section{INTRODUCTION}

Image Deblurring is one of the most important problems in image restoration. In this process, the observed image is modeled as the degradation of the sharp image, by convolution with blurring filter, plus some noise. Image deconvolution or deblurring has been widely used in the enhancement of images in photos and video cameras, in astronomy, in tomography, in remote sensing and in other biomedical imaging techniques.

Image Deblurring can be divided into two different types namely non-blind image deblurring and blind image deblurring. In non-blind image deblurring, the blurring filter is assumed to be known whereas in blind image deblurring both the image and the blurring operator are partially or totally unknown.

Due to the ill-conditioned nature of the blurring filter, the deblurring process is very difficult in non-blind image deblurring though the blurring operator is known. 
The International Journal of Multimedia \& Its Applications (IJMA) Vol.6, No.2, April 2014

Blind image deblurring is much more challenging when compared to non-blind image deblurring. Here the deblurring process leads to infinite number of solutions due to the presence of many pairs of blurring filters and image estimates. To solve this problem, most blind image deblurring methods restrict the type of blur filter based on either the use of regularizers [2], or through the use of parametric models [1], that are either through the soft way or hard way. This method aims to estimate the unknown blur from the observed blurred image and hence recover the original sharp image.

A recent iterative blind image deblurring method [7], uses a large regularization weight, which estimates the main features of the image. By decreasing the regularization parameter gradually, this method learns the image details. It requires manual stopping which is the main drawback. The methods developed for estimating the regularization parameter of non-blind image deblurring cannot be used for the blind case.

The already existing blind image deblurring methods require some tuning of the regularizing parameters. More work has been devoted to choose the accurate regularization parameter. The Discrepancy Principle (DP) [3] chooses the regularization parameter so that the variance of the residual matches that of the noise. Here the residual is the difference between the observed image and the blurred estimate. An extension of DP uses not only variance, but also the residual moments [4]. The method for automatically adjusting the regularization parameter for non-blind image deblurring is not the same for blind image deblurring. Stein's unbiased risk estimate (SURE) [5], [8], provides an estimate of the mean squared error (MSE), by assuming knowledge of the noise distribution and requiring an accurate estimate of its variance [6]. SURE-based approaches assume full knowledge of the degradation model and thus are not suitable for blind image deblurring. In blind image deblurring most of the existing methods require some tuning to obtain the regularization parameter.

In this paper, the iterative blind image deblurring method is based on the assumption that the additive noise is spectrally white. The residual associated with the deblurred image becomes white, if the blurring filter is correctly estimated, whereas with a wrong filter, the image estimate will exhibit structured artifacts which are not white. This method takes into account the prior information on the blurring filter. The estimation is guided to a good solution by first concentrating on the main edges of the image, and progressively dealing with smaller details. The proposed criterion yields significant results.

\section{BLIND IMAGE DEBLURRING METHOD}

Blind image deblurring method is used to deblur the degraded image, without prior knowledge of the blur kernel and additive noise.

The image degradation can be modeled as

$$
y=s * b+n
$$

where $\mathrm{y}$ is the degraded image, $\mathrm{s}$ is the sharp unknown image, $\mathrm{b}$ is the blur kernel and $\mathrm{n}$ is the noise involved. The deblurred method finds the cost function with respect to the sharp image $\mathrm{s}$ and the blur kernel $\mathrm{b}$ and is given by 
The International Journal of Multimedia \& Its Applications (IJMA) Vol.6, No.2, April 2014

$$
C(s, b)=\frac{1}{2}\|y-s * b\|^{2}+\alpha \phi(s)
$$

here the first term i.e., the data fidelity term results from assuming that the noise is white and Gaussian, $\alpha$ is the regularization parameter and $\Phi(\mathrm{s})$ is the regularizing function which contains prior information about s. Many image deblurring methods minimize the cost function (2) iteratively.

The step involved in blind image deblurring process is shown in Figure 1. The first step involved is to set the blur kernel $b$ to an identity operator and also to initialize the degraded image as the image estimate. In this deblurring process, the iteration starts with a high regularization value and is gradually decreased by multiplying it with a constant $\beta$ which is less than 1 . Too large values of $\alpha$ lead to cartoon-like images or over smoothed images while too small values leads to images influenced by more noise. Based on this, the new image estimate $\hat{s}$ and blur estimate $\hat{b}$ are formed. The stopping criterion of this blind deblurring method corresponds to setting the final value of the regularizing parameter which is based on the whiteness measures.

The cost function defined in (2) depends on the data fidelity term, the regularization value and the regularizing function $\Phi(\mathrm{s})$. The regularizer $\Phi(\mathrm{s})$ was chosen as it favors the sharp edges and also for certain values of the parameters, the prior is close to the actual observed edges. The sparse edges are quite visible in the first few iterations, but are almost unnoticeable in the final iteration. When the image to be deblurred is noisy, to prevent the appearance of the noise in the deblurred image, the final regularization cannot be made so weak.

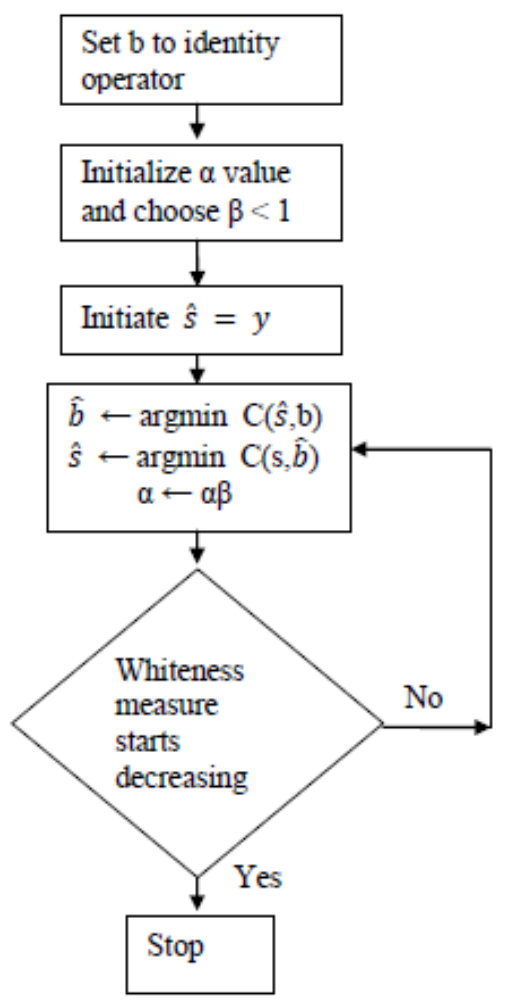

Figure 1. Process Involved in Blind Image Deblurring 
The International Journal of Multimedia \& Its Applications (IJMA) Vol.6, No.2, April 2014

The proposed method consists in selecting the regularization value or the final iteration of the algorithm that maximize the whiteness measure. This measure exhibits a clear peak and the method is stopped as soon as the whiteness measure starts to decrease.

\subsection{Edge Detection}

At the starting of the deblurring process, the estimate of the blur operator is poor and a strong regularization is required to make the edges sharper in the estimated image. During early iterations, only the main edge of the estimated image is identified. This is because of the high regularization value. The surviving sharp edges allow the estimate of the blurring operator to be improved. In the following iterations, the fainter edges are determined with the help of somewhat lower value of $\alpha$ and the improved filter. As the iteration increases, the smaller features are predicted with a gradual decrease of $\alpha$.

The edge detection filters in different orientations (Figure 2) are used for this prediction.
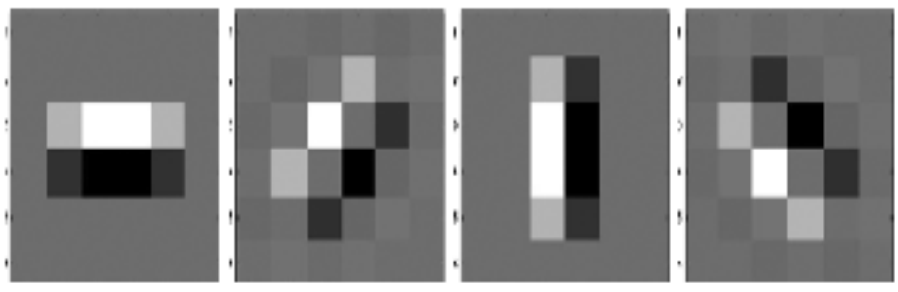

Figure 2. Edge Detection filters in the four orientations

Thus the sharp edges of the image estimate when compared with the blurred image, allows to learn and improve the estimate of the blurring filter and also to learn the finer image details.

\subsection{Whiteness Measures}

The selection of the stopping criteria are based on the measures of the fitness of the image estimate and the blur estimate. This is analysed based on the residual image given by

$$
r=y-\hat{s} * \hat{b}
$$

The nature of the residual is then compared with the additive noise $\mathrm{n}$. As this noise is assumed to be uncorrelated and spectrally white, the whiteness measure is used to access the adequacy of the image and the blur estimates. The residual image (3) is normalized to zero mean and unit variance. The auto-correlation of the normalized residual denoted by $r$ is given by

$$
R_{r r}(p, q)=\sum_{p, q} r(p, q) r(p-i, q-j)
$$

where the sum extends over the whole residual image.

The measure of whiteness of the residual image (3) is given as the distance between the autocorrelation (4) and the delta function. The delta function of the spectrally white image at the 
The International Journal of Multimedia \& Its Applications (IJMA) Vol.6, No.2, April 2014

origin is given by $\delta(\mathrm{p}, \mathrm{q})=1$, if $\mathrm{p}=\mathrm{q}=0$, Otherwise $\delta(\mathrm{p}, \mathrm{q})=0$. The delta function is the autocovariance of the spectrally white image.

The whiteness measure is the energy of auto-correlation beyond the origin given by

$$
M_{R}(r)=-\sum_{\substack{p, q=(-l,-l) \\ \neq(0,0)}}^{l, l}\left(R_{r r}(p, q)\right)^{2}
$$

Here the minus sign is used to make $\mathrm{M}_{\mathrm{R}}$ larger for whiter residuals and $l=4$.

\section{EXPERIMENTS AND RESULTS}

The benchmark images such as Lena, Barbara, and Cameraman are considered in this experiment. All images are of size $256 \times 256$ pixels. Blurs like out-of-focus and uniform blur are used; all are of size 9 × 9 (i.e. $21+1 \times 21+1$ ). Different blurred signal-to-noise-ratio (BSNR) like 30dB, $40 \mathrm{~dB}, 50 \mathrm{~dB}$ is used.

For evaluating the quality of the results, estimation of increase in signal to noise ratio (ISNR) is done. The residual image exhibit structures that are far from being spectrally white, for early iterations and also later iterations. For noisy blurred images, the estimated image quickly becomes degraded if the stopping point is chosen after the optimal point. For non-noisy blurred images, the choice of the stopping point has no influence after that point. At the best ISNR, the residual image has little structure, thus being approximately white and the auto-covariance is essentially a delta function. When the whiteness measure $\mathrm{M}_{R}(\mathrm{r})$ starts decreasing, the stopping criterion of the proposed method gets satisfied.

The test image (Fig. 3a) is blurred using a uniform blur filter with 30dB BSNR (Fig. 3b). The image estimate for the blurred image at different iterations and their respective blur estimates are shown in the figure 3. The final deblurred image (Fig. $3 \mathrm{~g}$ ) and its respective blur estimate (Fig. $3 \mathrm{~h}$ ) obtained show the effectiveness of the proposed method.

The ISNR values attained in the tests can be considered good, taking into account that the method under test is blind. The automatic stopping criteria show results that are slightly worse $(-0.38 \mathrm{~dB})$ than the best ISNR obtained by manual stopping, showing that the automatic criterion tends to stop earlier. Two filters namely uniform and out-of-focus blur for three different BSNR values are taken.

The ISNR values obtained are just slightly less than the ISNR values obtained by manual stopping in the proposed approach. 
The International Journal of Multimedia \& Its Applications (IJMA) Vol.6, No.2, April 2014

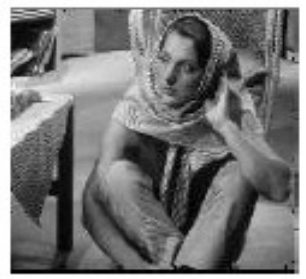

(a)

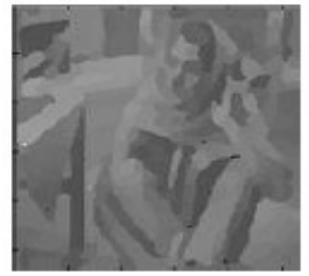

(c)

(d)

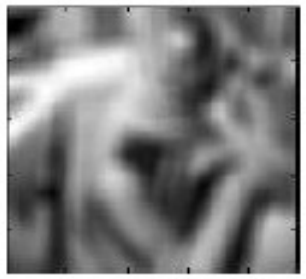

(b)

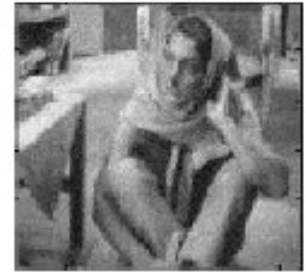

(e)

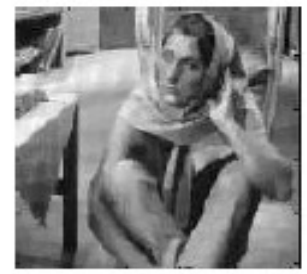

(g)

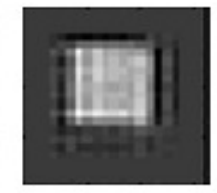

(h)

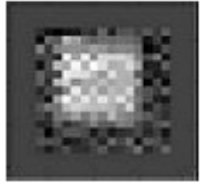

(f)

Figure. 3. Barbara image with uniform blur. (a) Sharp image (b) Image blurred with $30 \mathrm{~dB}$ noise (c)-(h) Images and the respective blur estimates in the iterative process

Table 1. ISNR values obtained for test images using different filters

\begin{tabular}{|c|c|c|c|c|}
\hline \multirow{2}{*}{ Filter } & \multirow{2}{*}{$\begin{array}{l}\text { BSNR } \\
\text { (dB) }\end{array}$} & \multicolumn{3}{|c|}{ ISNR (dB) } \\
\hline & & Barbara & Cameraman & Lena \\
\hline \multirow{3}{*}{ Uniform } & 30 & 5.03 & 5.22 & 5.26 \\
\hline & 40 & 6.11 & 6.96 & 7.08 \\
\hline & 50 & 8.11 & 9.95 & 8.07 \\
\hline \multirow{3}{*}{ Out-of-focus } & 30 & 5.66 & 5.31 & 5.83 \\
\hline & 40 & 6.86 & 7.91 & 7.84 \\
\hline & 50 & 9.27 & 11.15 & 10.79 \\
\hline
\end{tabular}


The International Journal of Multimedia \& Its Applications (IJMA) Vol.6, No.2, April 2014

Table 1 shows the increase in signal-to-noise ratio (ISNR) obtained by the proposed criterion for the different test images considered.

\section{CONCLUSIONS}

A new criterion has been proposed to select the regularization parameter and to stop iterative blind image deblurring method. Good estimates of both the image and the blurring operator are reached by initially considering the main edges of the image and then progressively handling the smaller ones. The method is based on the whiteness measures of the residual image. This approach does not require any knowledge about the convolution operator. Experimental tests on a variety of images degraded with out-of-focus and uniform blurs show good results. The ISNR values obtained from the automatic stopping criterion method are somewhat similar ( $0.38 \mathrm{~dB}$ less) to the values obtained by manual stopping.

\section{ACKNOWLEDGEMENTS}

I would like to thank my guide Dr.K.Bhoopathy Bagan for his guidance, constant encouragement and support. His extensive vision and creative thinking has been a source of inspiration throughout this project. And I also thank my review committee members for their valuable suggestions on improvement of this work.

\section{REFERENCES}

[1] J. P. Oliveira, M. A. T. Figueiredo, and J. M. Bioucas-Dias, "Blind estimation of motion blur parameters for image deconvolution,” in Proc. Iberian Conf. Pattern Recognit.. Image Anal., 2007, pp. 604-611.

[2] B. Amizic, S. D. Babacan, R. Molina, and A. K. Katsaggelos, "Sparse Bayesian blind image deconvolution with parameter estimation,” in Proc. Eur. Signal Process. Conf., 2010, pp. 626-630.

[3] A. M. Thompson, J. Brown, J. Kay, and D. Titterington, "A study of methods of choosing the smoothing parameter in image restoration by regularization," IEEE Trans. Pattern Anal. Mach. Intell., vol. 13, no. 4, pp. 326-339, Apr. 1991.

[4] L. Dascal, M. Zibulevsky, and R. Kimmel. (2008). Signal denoising by constraining the residual to be statistically noise-similar. Dept. Comput. Sci., Univ. Israel, Technion, Israel.

[5] Y. C. Eldar, "Generalized SURE for exponential families: Applications to regularization," IEEE Trans. Sig. Process., vol. 57, no. 2, pp. 471-481, Feb. 2009.

[6] X. Zhu and P. Milanfar, "Automatic parameter selection for denoising algorithms using a noreference measure of image content," IEEE Trans. Image Process., vol. 19, no. 12, pp. 3116-3132, Dec. 2010.

[7] M. S. C. Almeida and L. B. Almeida, "Blind and semi-blind deblurring of natural images," IEEE Trans. Image Process., vol. 19, no. 1, pp. 36-52, Jan 2010.

[8] R. Giryes, M. Elad, and Y. C. Eldar, "The projected GSURE for automatic parameter tuning in iterative shrinkage methods," Appl. Comput. Harmon. Anal., vol. 30, pp. 407-422, Jun. 2011.

[9] M. S. C. Almeida and L. B. Almeida, "Blind deblurring of natural images," in Proc. IEEE Int. Conf. Acoust., Speech, Signal Process., Apr. 2008, pp. 1261-1264. 